\title{
Chaotic Oscillations Type of Flexible Closed Cylindrical Nanoshells Under the Strip Loads Action*
}

\author{
Tatiana Yakovleva ${ }^{[0000-0003-3238-2317]}$, Michael Stasuk, and \\ Anton Krysko ${ }^{\text {[0000-0002-9389-5602] }}$ \\ Yuri Gagarin State Technical University of Saratov, Saratov, Russia \\ yan-tan1987@mail.ru, stasmikhail@yandex.ru, \\ anton.krysko@gmail.com
}

\begin{abstract}
The theory and data visualization of flexible closed cylindrical nanoshells nonlinear dynamics under the strip loads action are constructed. The theory is based on hypotheses: Kirchhoff-Love, modified couple stress theory, geometric non-linearity adopted in the T. von Karman form. To obtain the desired differential equations, the Hamilton-Ostrogradsky principle was used, which makes it possible to obtain the desired differential equations in mixed form describing nano effects. For reduction to the Cauchy problem in spatial coordinates, the Bubnov-Galerkin method in higher approximations is applied. Further, the Cauchy problem is solved by methods such as Runge-Kutta and Newmark. The convergence of the Bubnov-Galerkin method is studied depending on the number of terms in the original functions expansion in spatial coordinates. The oscillations transition scenario from harmonic to chaotic depending on the number of series members in the Bubnov-Garekin method, as well as depending on the type of load, geometric and size-dependent parameters, is investigated. The numerical experiment results were visualized by nonlinear dynamics methods and using wavelet analysis. It was revealed that the oscillations type substantially depends on these parameters; two types of chaos are observed: chaos and hyperchaos. This was revealed according to the chaos criterion given by Gulik, and the Lyapunov exponents study by the methods of Rosenstein, Kantz, and Wolf. A chaos type analysis was carried out based on the signs of Lyapunov exponents spectrum calculated by the Sano-Sawada method.
\end{abstract}

Keywords: Data Visualization, Flexible Closed Cylindrical Nanoshells, Modified Couple Stress Theory, Chaos, Hyperchaos, Lyapunov Exponents.

\section{$1 \quad$ Introduction}

The nonlinear dynamics problems visualization allows a qualitative assessment of the complex mechanical structures behavior at a new level. The modern technology constituent elements are subject to external dynamic action of a forceful nature. This fact

Copyright (C) 2020 for this paper by its authors. Use permitted under Creative Commons License Attribution 4.0 International (CC BY 4.0).

* This work was supported by the RFBR according to the research project № 20-08-00354. 
necessitates a comprehensive study of the structures behavior [1-3], determining their limiting states and identifying the type of chaotic oscillations. Previously, the chaotic oscillations type analysis was mainly considered in physics and radiophysics [4]. In mechanics, the type of chaotic state was studied using classical systems as an example [5-6]. An important issue is the methods of the results scientific visualization, which allow obtaining reliable information about the ongoing process. This article is aimed at constructing a theory and data visualization for the chaotic oscillations analysis of flexible closed cylindrical nanoshells under the strip loads action.

\section{Mathematical Model of a Cylindrical Nanoshell}

The study object is a closed cylindrical shell, occupying a region $\Omega=\{x, y, z \mid(x, y, z) \in[0 ; L] \times[0 ; 2 \pi] \times[-h ; h]\}$ in the space $\mathrm{R}^{3}$, where $L$ and $h$ - are shell length and thickness. The coordinate system is entered as shown in Fig. 1.

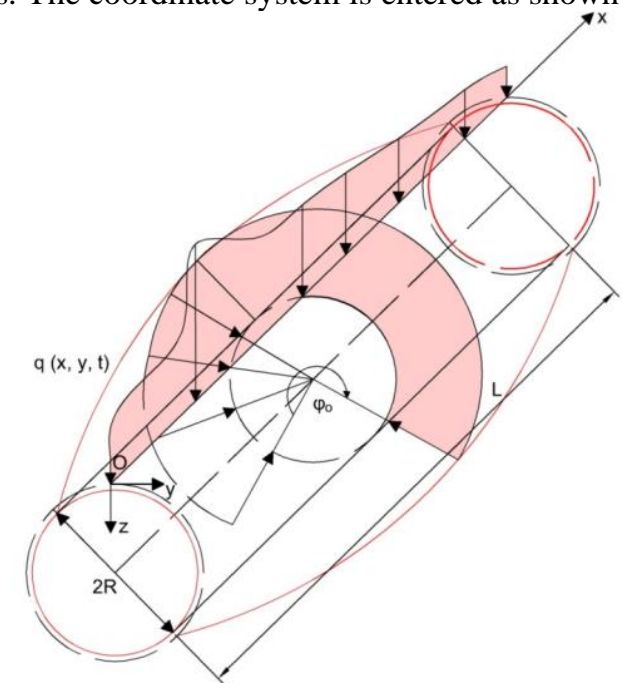

Fig. 1. Scheme of closed cylindrical nanoshell.

The shell is isotropic, homogeneous, elastic and obeys Hooke's law. Geometric nonlinearity is taken into account according to the T. Karman model. The direct normals hypothesis is valid (Kirchhoff-Love hypothesis). Given the modified couple stress theory [7-8], we obtain the resolving equations of shell motion, boundary and initial conditions from the Ostrogradsky-Hamilton variation principle. According to this principle, close movements are compared that bring the system of material points from the initial position at time $t_{0}$ to the final position at time $t_{1}$. For true movements, the condition must be satisfied: 
Here $K$ - kinetic energy, $U$ - potential energy, $W$ - external work related to distributed forces and energy dissipation.

Using the standard procedure of the variations calculus, we obtain the shell motion equations and the deformations compatibility equation in the form:

$$
\left\{\begin{array}{l}
D \Delta^{2} w-k y \frac{\partial^{2} F}{\partial x^{2}}-L(w, F)+\frac{\partial^{2} w}{\partial t^{2}}+\varepsilon \frac{\partial w}{\partial t}=q(x, y, t) \\
\frac{1}{E h} \Delta^{2} F=-\frac{1}{2} L(w, w)-k_{y} \frac{\partial^{2} w}{\partial x^{2}}
\end{array}\right.
$$

where $D=\frac{E h^{3}}{12\left(1-v^{2}\right)}+\frac{E h \gamma^{2}}{2(1+v)}, \quad \Delta^{2}(\cdot)=\frac{\partial^{4}(\cdot)}{\partial x^{4}}+2 \frac{\partial^{4}(\cdot)}{\partial x^{2} \partial y^{2}}+\frac{\partial^{4}(\cdot)}{\partial y^{4}}$

$L(w, F)=\frac{\partial^{2} w}{\partial x^{2}} \frac{\partial^{2} F}{\partial y^{2}}+\frac{\partial^{2} w}{\partial y^{2}} \frac{\partial^{2} F}{\partial x^{2}}-2 \frac{\partial^{2} w}{\partial x \partial y} \frac{\partial^{2} F}{\partial x \partial y}, \quad L(w, w)=2\left[\frac{\partial^{2} w}{\partial x^{2}} \frac{\partial^{2} w}{\partial y^{2}}-\left(\frac{\partial^{2} w}{\partial x \partial y}\right)^{2}\right]-$ are known nonlinear operators.

We attach to the system (2) the boundary conditions for articulated support at the ends:

$$
w=0 ; \frac{\partial^{2} w}{\partial x^{2}}=0 ; F=0 ; \frac{\partial^{2} F}{\partial x^{2}}=0 \text { at } x=0 ; 1,
$$

and periodicity condition at $\mathrm{y}=0 ; 2 \pi$.

In addition to the boundary conditions, we add to the equations (2) the initial conditions:

$$
\left.w\right|_{t=0}=0,\left.\dot{w}\right|_{t=0}=0
$$

Equations (2-4) are reduced to dimensionless form using the following parameters: $w=h \bar{w}, F=E h^{2} \bar{F}, t=t_{0} \bar{t}, \varepsilon=\bar{\varepsilon} / \tau, x=L \bar{x}, y=R \bar{y}, k_{y}=\bar{k}_{y} \frac{h}{R^{2}}, q=\bar{q} \frac{E h^{4}}{L^{2} R^{2}} \quad \tau=\frac{L R}{h} \sqrt{\frac{\rho}{E g}}$, $\lambda=\frac{L}{R}$, where $L$ and $R=R_{y}$ - are shell length and radius. Here $t$ - time, $\varepsilon$ - resistance coefficient of the medium in which the shell moves, $v=0.3$ - Poisson's ratio, $E$ - Young's modulus, $g$ - acceleration of gravit, $\rho$ - material density, $\gamma$ - sizedependent coefficient, $q(x, y, t)$ - strip load. The line over dimensionless quantities is omitted for simplicity.

In this paper, the chaotic oscillations type of a closed cylindrical size-dependent nano-shell with pivotally fixed edges is first studied. A transverse distributed strip alternating load with amplitude $q_{0}$ acts on the shell (see Fig. 2). 


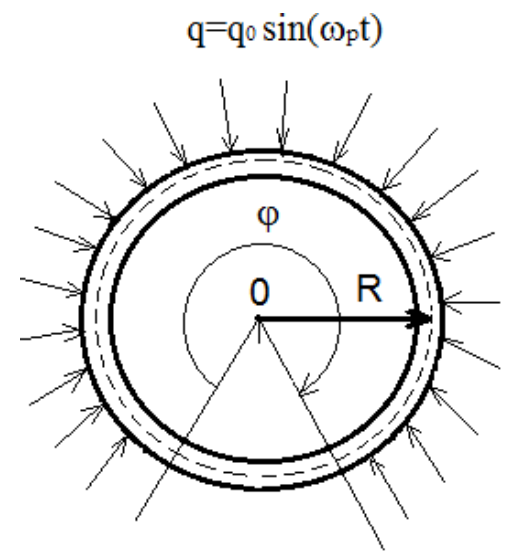

Fig. 2. Shell loading diagram.

\section{Solution Methods}

Systems of nonlinear partial differential equations are reduced to a system of ordinary differential equations by the Bubnov-Galerkin method in higher approximations. The Cauchy problem is solved by the Runge-Kutta method of the fourth accuracy order and Newmark method.

Further visualization of the closed cylindrical size-dependent shell oscillations nature study was carried out by nonlinear dynamics methods with the $w$ signals construction at the center of the applied shell load, phase portraits, Fourier power spectra and using wavelet analysis. For the data visualization reliability, the Morlet, Gauss 8 Gauss 32, and Haar wavelets were used as the mother wavelet. Methods and approaches of nonlinear dynamics find their application in various branches of modern science, in mechanics, history [9], in the analysis of EEG data (time series) [10], etc. An analysis of the nanoshell chaotic oscillations type is carried out according to the chaos criterion given by Gulik [11], as well as on the basis of the Lyapunov exponents spectrum signs calculating by the Sano-Sawada method [12].To confirm the reliability of the results obtained, the senior Lyapunov indices are calculated by several methods: Wolf [13], Kantz [14], Rosenstein [15] and Sano-Sawada. The results visualization was carried out using the software packages MATLAB and Mathcad.

\section{$4 \quad$ Numeric Results}

The shell is affected by a transverse distributed strip alternating load with amplitude $q_{0}$, natural vibration frequency $\omega_{p}=20.3$, load angle $\varphi_{0}=5.98$ along the entire length of the cylinder. In the Bubnov-Galerkin method, the series members number was taken equal to $N=5,7,9,11,13,15$ for each of the size-dependent coefficient values $\gamma=0,0.1,0.3,0.5,0.7$. 
Chaotic Oscillations Type of Flexible Closed Cylindrical Nanoshells Under the Strip Loads ... 5

Table 1. The dynamic characteristics of a cylindrical shell at $\lambda=1, q_{0}=0.18575, N=15$.

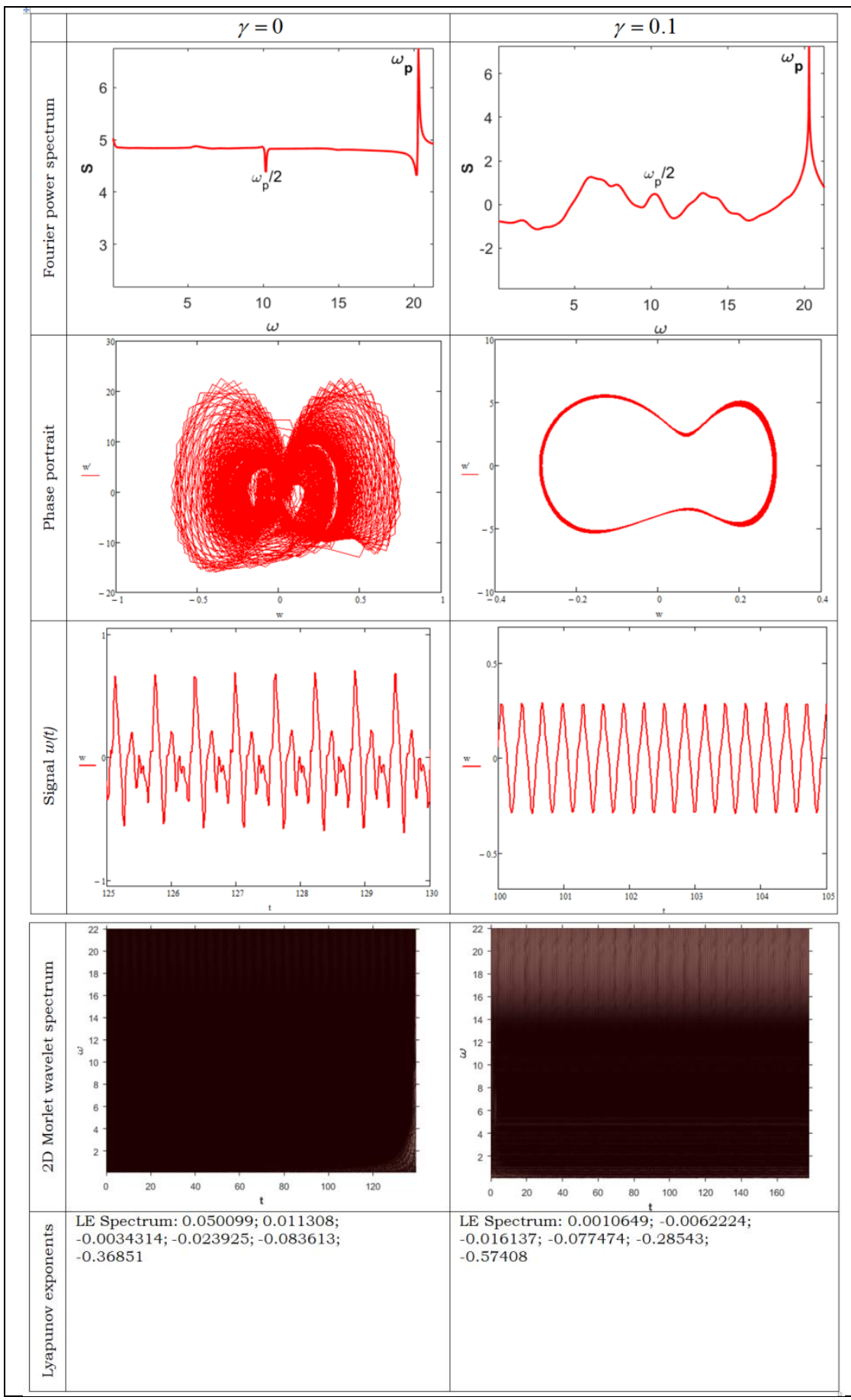


Table 1 presents the oscillations graphs of the nano shell for the following parameters values $\lambda=1, q_{0}=0.18575, N=15, \gamma=0,0.1$.

In the case of a macro-sized ( $\gamma=0$ ) cylindrical shell, in addition to the driving oscillations frequency $\omega_{\mathrm{p}}$, the Hopf bifurcation at $\omega_{p} / 2$ is observed in the Fourier power spectrum. In the Lyapunov exponents spectrum, we have positive senior and positive second Lyapunov exponents, while the third or sixth exponents have negative values. This means that the oscillatory system is in a state of hyper chaos [5] (Table 1).

In the case of a nano scale $(\gamma=0.1)$ cylindrical shell, in addition to the driving oscillations frequency $\omega_{\mathrm{p}}$, the Hopf bifurcation $\omega_{\mathrm{p}} / 2$ and a number of frequencies are observed in the Fourier power spectrum. In the Lyapunov exponents spectrum we have a positive senior exponent, while the other five exponents are negative. This indicates the chaotic nature of shell vibrations [5] (Table 1).

Table 2. The oscillation types of the cylindrical shell.

\begin{tabular}{|c|c|c|c|}
\hline \multicolumn{4}{|c|}{$\lambda=1, q_{0}=0.18575$} \\
\hline \multicolumn{2}{|l|}{$\gamma=0$} & \multirow{2}{*}{$\begin{array}{l}\gamma=0.1 \\
: 0 \ldots-\ldots\end{array}$} & \multirow{2}{*}{$\begin{array}{l}\gamma=0.3-0 . \\
: 0-\cdots-\end{array}$} \\
\hline$N=5:$ & $\ldots \ldots$ & & \\
\hline$N=7:$ & $0 \ldots$ & : $\ldots \ldots$ & : $0 \ldots$ \\
\hline$N=9:$ & $++\ldots$ & $0 \ldots$ & : $0 \ldots$ \\
\hline$N=11:$ & $++\ldots$ & $0 \ldots$ & : $0 \ldots$ \\
\hline$N=13:$ & $00 \ldots$ & 0 & : $0 \ldots$ \\
\hline$N=15:$ & $++\ldots$ & : $\ldots \ldots$ & : $0 \ldots \ldots$ \\
\hline \multicolumn{4}{|c|}{$\lambda=3, q_{0}=0.18575$} \\
\hline \multicolumn{2}{|l|}{$\gamma=0$} & $\gamma=0.1-0.3$ & $\gamma=0.7$ \\
\hline$N=5:$ & $++\ldots$ & :+ +... & $: 0 \ldots$ \\
\hline$N=7:$ & $+\ldots .$. & $:+\ldots$. & $:+\ldots \ldots$ \\
\hline$N=9:$ & $+\ldots .$. & $:+\ldots$ & $:+\ldots \ldots$ \\
\hline $\mathbf{N}=11:$ & $0 \ldots$ & $:+\ldots$ & $:+\ldots$. \\
\hline $\mathbf{N}=13:$ & $+\ldots .$. & : + . . - & : $+\ldots$ \\
\hline $\mathbf{N}=\mathbf{1 5}$ & $+\ldots .$. & : $+\ldots$ & : $0 \ldots \ldots$ \\
\hline $0 \ldots$ & -. $\mathrm{Ha}$ & $1 \mathrm{~s} ;+\ldots .$. & Hyper ch \\
\hline
\end{tabular}

In the case of the size-dependent parameter $\gamma$, at the previous remaining parameters values the cylindrical nanoshell performs harmonic oscillations, since the senior Lyapunov exponent has a value close to zero, and the remaining five exponents in the spectrum have negative values [5] (Table 2).

Table 2 presents the visualization results of the chaotic oscillations type analysis for a closed cylindrical size-dependent shell depending on the geometric parameter ( $\lambda=1$ and $\lambda=3)$, size-dependent parameter $(\gamma=0,0.1,0.3,0.5,0.7)$, series members number in the Bubnov-Galerkin method $(\mathrm{N}=5,7,9,11,13,15)$. The each sign of the 
first six Lyapunov exponents in the spectrum is indicated. For $\lambda=1$, with an increase in the size-dependent parameter $\gamma$, chaotic oscillations transform into harmonic ones. At $\lambda=3$ such a process is observed only at $N=5,15$.

\section{Conclusion}

In the work, by visualizing scientific data, a chaotic oscillations type analysis of flexible closed cylindrical nanoshells under the strip loads action is carried out. The scientific visualization of the results is based on the construction of signals, phase portraits, Fourier power spectra and the use of wavelet transforms. It has been established that the Morlet wavelet is the most informative for this class of problems, since it gives the best frequency localization at every moment in time. It is worth noting that the Fourier power spectrum gives a general picture of the nature of the oscillations of nanoshells over the entire time interval. The proposed approach allows us to study the nonlinear dynamics of the flexible closed cylindrical nanoshells, under the influence of an external alternating load, depending on the size-dependent coefficient. It was revealed that the oscillations type significantly depends on the amplitude and frequency of the load, size-dependent and geometric parameters, as well as on the members number of series in the Bubnov-Galerkin method. The cylindrical shell can perform harmonic or chaotic oscillations, while depending on the number of positive Lyapunov exponents, two types of chaos are observed in the spectrum: chaos and hyperchaos.

\section{References}

1. Krysko-Jr. V., Awrejcewicz J., Yakovleva T., Kirichenko A., Szymanowska O., Krysko V.: Mathematical modeling of MEMS elements subjected to external forces, temperature and noise, taking account of coupling of temperature and deformation fields as well as a nonhomogenous material structure. Communications in Nonlinear Science and Numerical Simulation. Vol. 72. 39-58. 2019. https://doi.org/10.1016/j.cnsns.2018.12.001.

2. Yakovleva T., Krysko-jr. V., Krysko V.: Nonlinear dynamics of the contact interaction of a three-layer plate-beam nanostructure in a white noise field. Journal of Physics: Conference Series. 1210 (2019) 012160. https://iopscience.iop.org/article/10.1088/1742$6596 / 1210 / 1 / 012160$.

3. KryskoV., Yakovleva T., Papkova I., Saltykova O., Pavlov S.: The contact interaction of size-dependent and multimodulus rectangular plate and beam. Journal of Physics: Conf. Series $1158 \quad$ (2019) 032021. https://iopscience.iop.org/article/10.1088/17426596/1158/3/032021.

4. Shurygina S.: On the behavior of one of the positive Lyapunov exponent in mutually coupled chaotic oscillators. Papers from the conference for young scientists "Presenting Academic Achievements to the World". 2010. P. 129-132.

5. Awrejcewicz J., Krysko A., Erofeev N., Dobriyan V., Barulina M., Krysko V.: Quantifying Chaos by Various Computational Methods. Part 1: Simple Systems. Entropy in Dynamic Systems. 124-137, 2018.

6. Awrejcewicz J., Krysko A., Kutepov I., Zagniboroda N., Dobriyan V., Krysko V.: Chaotic dynamics of flexible euler-bernoulli beams. Chaos, 2013. 
7. Mindlin R., Tiersten H. Effects of couple-stresses in linear elasticity. Archive for Rational Mechanics and Analysis. 11 415-448. (1962).

8. J. Yang, T. Ono and M. Esashi, Energy dissipation in submicron thick single-crystal silicon cantilevers, J. Microelectromech. Sys. 11(6), 775-783 (2002).

9. Yaroshenko T.Y., Krysko D.V., Dobriyan V., Zhigalov M.V., Vos H., Vandenabeele P., Krysko V.A. Wavelet Modeling and Prediction of the Stability of States: The Roman Empire and the European Union. Communications in Nonlinear Science and Numerical Simulation 2015. 26 (1-3): 265-275.

10. Kutepov I.E., Krysko A.V., Dobriyan V.V., Yakovleva T.V., Krylova E.Yu., Krysko V.A. Visualization of EEG signal entropy in schizophrenia. Scientific Visualization, 2020, vol. 12, n. 1, p. $1-9$.

11. Gulick D.: Encounters with chaos. New York: McGraw-Hill. 1992.

12. Sano, M., Sawada, Y.: Measurement of Lyapunov spectrum from a chaotic time series. Phys. Rev. Lett. 55, 1082 (1985).

13. Wolf A, Swift JB, Swinney HL, Vastano JA. Determining Lyapunov exponents from a time series. Physica. 1985, 16D, pp. 285-317.

14. Kantz, H. (1994).A robust method to estimate the maximal Lyapunov exponent of a time series. Physics letters A, 185(1), 77-87.

15. Rosenstein, M.T., Collins, J.J., de Luca, C.J.: A practical method for calculating the largest Lyapunov exponent from small data sets. Physica D 65, 117 (1993). 